difficult the labour the greater the arousal or "stress" to the baby. (Whether this was a good or bad thing was conjectural.)

A similar increase in conductance was also seen immediately after an infant was subjected to a heel stab to obtain a blood sample. This plus simple observation of infants in special care baby units convinced him that infants did experience pain and distress even though their responses were obviously limited.

Mention of inflicting pain on neonates introduced what was unquestionably the most clinically relevant topic of the day. Dr K J S Anand, a research fellow from the John Radcliffe Hospital, Oxford, showed that babies who undergo major surgery show large increases in a variety of different metabolic and hormonal variables. Furthermore, those infants who have surgery carried out under minimal anaesthesia show much greater rises than those who are given potent anaesthesia.

Clearly it came as a surprise to some of the audience to hear that some surgery and certain invasive procedures in neonates are carried out with only minimal anaesthesia and analgesia in some hospitals in Britain, Europe, and America. This is done on the assumption that because preterm and even term infants have no memory of pain they are probably not capable of discriminating painful from other stimuli and because anaesthetic agents may have adverse effects on the cardiovascular and respiratory systems they are best avoided. Dr Anand said that as a result of his work the paediatric anaesthetists in Oxford have changed their practice and now ensure that the babies have fully adequate anaesthesia and analgesia. They also now give more postoperative analgesia. One or two members of the audience voiced their concern that this stress at or soon after birth may have long term psychological sequelae.

There seems no doubt that the neonate and possibly the fetus is capable of "feeling" considerably more than is widely appreciated. It is to be hoped that Dr Chiswick's suggestion that those who work in special care baby units should get together and review their policies on management is promptly taken up.

TESSA RICHARDS

Assistant Editor,

$B M \mathcal{F}$

\section{National Health Service: control of nursing manpower}

Whether or not the National Health Service is "safe in its hands," a government committed to a reduction in public expenditure might be expected to look critically at nursing manpower. In the year 1983-4 nurses' salaries cost $£ 3487$ million, accounting for $34 \%$ of the NHS budget - and nearly $3 \%$ of all public expenditure. ${ }^{1}$ Nursing manpower in this context includes all grades of qualified staff, learners (pupil and student nurses), and unqualified staff (nursing auxiliaries). Nursing auxiliaries are quite properly included in the nursing budget, but it is unfortunate that yet another government document classifies them as "nurses." Not only does this put a group with little or (in most cases) no training on the same level as those who have undergone training but it also leads the public to think that they, and their relatives, are being cared for by qualified staff.
One of the claims of the present government is that expenditure on nursing manpower has increased during its term of office. Certainly between 1976 and 1983 there was a $16 \%$ increase in the bill for nursing salaries in England, with similar increases in Scotland and Wales. In real terms, however, this figure falls to $9 \%$ when allowance is made for the reduction in working hours to $371 / 2$ a week in 1980 . Furthermore, in England between 1978 and 1983 the number of hospital patients increased by roughly $12 \%$ and outpatient attendances increased by $7 \%$. Turnover in the acute sector has speeded up, meaning that patients in these beds require more nursing care, as do the patients aged 65 and over, who now make up over one quarter of all acute admissions. Technological innovations and the need for periods of postregistration training to enable nurses to work in specialist areas have reduced the hours available for direct patient care. Hence the claim that increased numbers have improved care has to be viewed with some scepticism. Finally, while numbers of nurses in deprived parts of the NHS, such as those caring for the aged, mentally ill, and handicapped, have increased, their numbers started from a pitifully low base.

Against that background a report published this summer from the Comptroller and Auditor General has shown that in most health authorities the planning and control of nurse manpower is haphazard. Various methods are available for estimating nursing manpower needs, either "topdown" (that is, by the use of measures of output and activity) or "bottom up" (that is, by the use of professional nursing judgment about the number of nurses required to provide care). In practice, most authorities use either a historical base or one swayed by the availability of resources to determine how many nurses to employ. Few seem to have made any serious attempt to consider the most suitable "mix" of grades of staff in relation to the type of patient or aspect requiring care. Some authorities are now attempting to develop performance indicators, which may help, and the DHSS has just announced a group to review "skill mix," but much more effort is needed.

The report suggests several ways in which substantial savings might be made. The first of these is the time allowed for shift overlap-which in the sample studied varied from two to six hours. The National Audit Office report considers 90 minutes (to include a meal break) to be adequate, and its appendix 9 shows the money that could be recouped from a reduction in this time. Clearly the suggestions warrant careful study, but account needs to be taken of what is done by nurses in that overlap period. In some cases the extra hands are needed to get heavy patients up and dressed; in others the time is used for teaching learners. No doubt some rosters might be altered, but local circumstances (such as availability of public transport, especially late at night) may be a hindering factor. Wherever savings are being proposed staff organisations will want assurances that the attempt to save money will not disadvantage either the patients or the nurses.

Another comment made in the report is that in some hospitals staff were employed on a grade higher than that needed by the task. The explanation seemed to be the lack of suitably qualified staff. Again the implications for the care of patients might be sinister: the cheap solution is for authorities to employ untrained staff in these circumstances, but that temptation should be resisted.

The study uncovered the fact that nurses were still carrying out work that could be considered "non-nursing" and that elimination of these tasks would produce a 
considerable financial saving. Any strict definition of "nonnursing" duty is difficult; nurses would claim that any aspect of care, including serving meals, which directly affects the wellbeing of the patient is a nursing responsibility. A more fruitful line of study would be the time spent by nurses doing the work of absent medical or paramedical staff, or both. Since nurses provide 24 hour, seven day a week cover in hospitals they often find themselves "filling the gap" left by many other grades from domestic to medical staff.

One of the problems well known to all nurse managers and highlighted by the report is (for a variety of reasons) the staffing by nurses of empty beds. The report reaffirms the practicability of five day wards for many types of acute and minor surgery. The savings made would mainly come from reductions in payments for unsocial hours-but staff organisations commonly claim that only by these payments can many of their members earn an adequate take home wage.

Many of the savings suggested by the report require collaboration from other groups, not least medical staff. While financial savings may be possible in nursing manpower, care must be taken to ensure that expenditure elsewhere does not have to rise and that standards of nursing care do not suffer.

Christine M Chapman

Professor of Nursing Studies,

University of Wales College of Medicine,

Cardiff CF4 4XN

1 Comptroller and Auditor General. National Health Service: control of nursing manpower. London: HMSO, 1985

\section{The human face}

Our lives are the richer for the wealth of facial expression of which we are capable-from a deep blush to a smile like a glint of winter sunshine on the brass handle of a coffin. For most neonates, children, and adults the face gives an identity, an appearance of intelligence, and a means of communicating emotion. Children with facial agenesis are regarded as unintelligent. Down's syndrome is made more acceptable by plastic surgery. Unrecognised facial rigidity in early parkinsonism results in misread emotions. Schizophrenics are limited in their ability to interpret facial expression. ' Lip reading depends on facial expression as well as lip movement and is difficult if the speaker is hirsute or wears dark glasses.

Any discussion of the origin or triggering of facial expression produces complex theoretical arguments. Is the expression of emotion determined centrally? Some facial acts-for example, sneezing, coughing, and yawning-are not associated with emotion. Blushing and pallor are, but these are involuntary and produce relief of tension. The role of peripheral stimuli in the generation of emotional feeling forms the basis of the James-Lange hypothesis, ${ }^{2}$ which, as modified by Schachter, ${ }^{3}$ suggests that undifferentiated visceral stimuli or arousal of the sympathetic nervous system combine with cognitive and social factors to pattern the emotional experience. The evidence is conflicting. Stimulation of the relevant visceral centres within the brain might produce similar gastrointestinal reactions. ${ }^{4}$ Laughter may be precipitated by central lesions. ${ }^{5}$ Emotional lability occurs with bulbar palsy when emotional feelings may bear little relation to facial expressions. Hohmann interviewed fellow paraplegics and found a decrease in emotional feelings, although overtly emotional behaviour continued to be displayed. ${ }^{6}$ In the Riley-Day syndrome of familial dysautonomia emotional instability is associated with attacks of severe vomiting. ${ }^{7}$

It is possible that facial manifestations of emotion are not the terminal event in a causal sequence but may provide information by muscular feedback ${ }^{8}$ and influence heart rate and other autonomic activities. ${ }^{9}$ If emotions are expressed freely they are intensified-repression may soften them. ${ }^{10}$

Darwin recognised that facial expression of emotion was universal to all cultures. ${ }^{11}$ He considered that this expression had evolved as an adaptive, serviceable habit to communicate information about probable future behaviour. ${ }^{12}$ Certain expressions such as grinning developed as responses for the protection of vulnerable areas, ${ }^{12}$ others had an antithetical action reversing the emotion to signify friendship. ${ }^{11}$ But why should humans, who have such a powerful means of communication, notably language, be the one animal to develop such a complex repertoire of facial movements? And what purpose might be served by displaying facial expressions of fear to an enemy, or surprise to an intruder ${ }^{23}$ Ontogenically not all facial expressions begin as reflex phenomena. The earliest smiles, best seen in premature babies, occur during sleep in the absence of known stimuli and are associated with low, oscillating states of excitement originating in the brain stem or limbic system. The first waking smile occurs when the infant is satiated after feeding. Alert responses to tactile stimulation, to voices, or to perpetuate a novel situation come later. Laughter is initially provoked by vigorous physical stimulation long before its use as a reaction to socially meaningful auditory or visual experiences. ${ }^{14}$

To Waynbaum, all emotional reactions produced circulatory changes and entailed mobilising or reducing energy demands. ${ }^{15}$ Blushing, sobbing, laughing, and frowning are accompanied by contractions of the diaphragm, and parts of the face are suffused even in smiling. Voluntary and spontaneous emotional expressions are controlled by different neural pathways, but all emotional acts are associated with compression of facial veins and arteries by the facial muscles pushing against the bony structure and acting as tourniquets regulating blood flow. Furthermore, the facial artery, a major branch of the external carotid, is rich in muscular tissue and by diverting blood to the face may maintain a constant supply of blood to the brain even if the rest of the circulation undergoes violent variations. That the external carotid blood flow may modify the very precise autoregulation of cerebral blood flow is unlikely; but the venous return might be regulated by alterations of the facial musculature. Of greater importance, however, may be the connection between emotional reactions and brain temperature. ${ }^{13}$ Zojonc surmises that there is a narrow optimum temperature range for the synthesis of neurotransmitters and that brief fluctuations may cause their release. ${ }^{13}$ The temperature of the mammalian brain is controlled by three mechanisms: brain metabolism, cerebral blood flow, and the temperature within the internal carotid artery. In lower mammals the internal carotid artery is cooled below body core temperature by a network of medium sized arteries from the external carotid artery. In mammals with no such vascular plexus the internal carotid artery runs through the cavernous sinus which receives venous blood draining the nasal mucosa and the skin of the face. Moreover, blood flow in the angularis oculi vein, which 